\title{
Discovery of plasma messenger RNA as novel biomarker for gastric cancer identified through bioinformatics analysis and clinical validation
}

\author{
Wei Cao ${ }^{\text {Equal first author, 1, 2, } 3}$, Dan Zhou ${ }^{\text {Equal first author, 1, 2, } 3 \text {, Weiwei Tang }}{ }^{4}$, Hanxiang An ${ }^{\text {Corresp., 1, } 4}$, Yun Zhang ${ }^{\text {Corresp. 1, 2, } 3}$ \\ ${ }^{1}$ Department of Medical Oncology, Xiang'an Hospital of Xiamen University, School of Medicine, Xiamen University, Xiamen, Fujian, China \\ 2 Key Laboratory of Design and Assembly of Functional Nanostructures, Fujian Provincial Key Laboratory of Nanomaterials, Fujian Institute of Research on \\ the Structure of Matter, Chinese Academy of Sciences, Fuzhou, China \\ 3 Department of Translational Medicine, Xiamen Institute of Rare Earth Materials, Chinese Academy of Sciences, Xiamen, China \\ 4 Department of Medical Oncology, Cancer Hospital, The First Affiliated Hospital of Xiamen University, School of Medicine, Xiamen University, Teaching \\ Hospital of Fujian Medical University, Xiamen, Fujian, China \\ Corresponding Authors: Hanxiang An, Yun Zhang \\ Email address: an_hanxiang@126.com, zhangyunfjirsm@163.com
}

Background. Gastric cancer (GC) is the third leading cause of cancer-related death worldwide, partially due to lack of effective screening strategies. Thus, there is a stringent need for non-invasive biomarkers to improve patient diagnostic efficiency in GC.

Methods. This study initially filtered messenger RNAs (mRNAs) as prospective biomarkers through bioinformatics analysis. Clinical validation was conducted using circulating mRNA in plasma from patients with GC. Relationships between expression levels of target genes and clinicopathological characteristics were calculated. Then, associations of these selected biomarkers with overall survival (OS) were analyzed using the Kaplan-Meier plotter online tool. Results. Based on comprehensive analysis of transcriptional expression profiles across 5 microarrays, top 3 over- and underexpressed mRNAs in GC were generated. Compared with normal controls, expression levels of collagen type VI alpha 3 chain (COL6A3), serpin family H member 1 (SERPINH1) and pleckstrin homology and RhoGEF domain containing G1 (PLEKHG1) were significantly upregulated in GC plasmas. Receiver-operating characteristic (ROC) curves on the diagnostic efficacy of plasma COL6A3, SERPINH1 and PLEKHG1 mRNAs in GC showed that the area under the ROC (AUC) was $0.720,0.698$ and 0.833 , respectively. Combined these three biomarkers showed an elevated AUC of 0.907 . Interestingly, the higher COL6A3 level was significantly correlated with lymph node metastasis and poor prognosis in GC patients. High level of SERPINH1 mRNA expression was correlated with advanced age, poor differentiation and lower OS and PLEKHGI was also associated with poor OS in GC patients. Conclusion. Our results suggested that circulating COL6A3, SERPINH1 and PLEKHG1 mRNAs could be putative noninvasive biomarkers for $\mathrm{GC}$ diagnosis and prognosis. 
1 Discovery of plasma messenger RNA as novel

2 biomarker for gastric cancer identified through

3 bioinformatics analysis and clinical validation

4

5 Wei Cao ${ }^{1,2,3,5}$, Dan Zhou ${ }^{1,2,3,5}$, Weiwei Tang ${ }^{4}$, Hanxiang An ${ }^{1,4}$ and Yun Zhang ${ }^{1,2,3}$

6

$7{ }^{1}$ Department of Medical Oncology, Xiang'an Hospital of Xiamen University, School of

8 Medicine, Xiamen University Xiamen, Fujian, China;

$9{ }^{2}$ Key Laboratory of Design and Assembly of Functional Nanostructures, Fujian Provincial Key

10 Laboratory of Nanomaterials, Fujian Institute of Research on the Structure of Matter, Chinese

11 Academy of Sciences, Fuzhou, China;

$12{ }^{3}$ Department of Translational Medicine, Xiamen Institute of Rare Earth Materials, Chinese

13 Academy of Sciences, Xiamen, Fujian, China;

$14{ }^{4}$ Department of Medical Oncology, Cancer Hospital, The First Affiliated Hospital of Xiamen

15 University, School of Medicine, Xiamen University, Teaching Hospital of Fujian Medical

16 University, Xiamen, Fujian, China

$17{ }^{5}$ These authors contributed equally to this work.

18

19 Corresponding Author:

20

Han-Xiang An

21

22

23

24

25

Department of Medical Oncology, Xiang'an Hospital of Xiamen University, Xiamen, Fujian, China

Email address: anhanxiang@xmu.edu.cn

Yun Zhang

Xiamen Institute of Rare Earth Materials, No.258 Xiheng Duishan Road, Ji-mei District, Xiamen, Fujian, 361021, China

7 Email address: zhangy@fjirsm.ac.cn 


\section{Abstract}

Background. Gastric cancer (GC) is the third leading cause of cancer-related death worldwide, partially due to lack of effective screening strategies. Thus, there is a stringent need for noninvasive biomarkers to improve patient diagnostic efficiency in GC.

Methods. This study initially filtered messenger RNAs (mRNAs) as prospective biomarkers through bioinformatics analysis. Clinical validation was conducted using circulating mRNA in plasma from patients with GC. Relationships between expression levels of target genes and clinicopathological characteristics were calculated. Then, associations of these selected biomarkers with overall survival (OS) were analyzed using the Kaplan-Meier plotter online tool.

Results. Based on comprehensive analysis of transcriptional expression profiles across 5 microarrays, top 3 over- and underexpressed mRNAs in GC were generated. Compared with normal controls, expression levels of collagen type VI alpha 3 chain (COL6A3), serpin family $H$ member 1 (SERPINH1) and pleckstrin homology and RhoGEF domain containing G1 (PLEKHG1) were significantly upregulated in GC plasmas. Receiver-operating characteristic (ROC) curves on the diagnostic efficacy of plasma COL6A3, SERPINH1 and PLEKHG1 mRNAs in GC showed that the area under the ROC (AUC) was 0.720, 0.698 and 0.833, respectively. Combined these three biomarkers showed an elevated AUC of 0.907. Interestingly, the higher COL6A3 level was significantly correlated with lymph node metastasis and poor prognosis in GC patients. High level of SERPINH1 mRNA expression was correlated with advanced age, poor differentiation and lower OS and PLEKHG1 was also associated with poor OS in GC patients.

Conclusion. Our results suggested that circulating COL6A3, SERPINH1 and PLEKHG1 mRNAs could be putative noninvasive biomarkers for $\mathrm{GC}$ diagnosis and prognosis.

\section{Introduction}

Gastric cancer (GC) is one of the most common diagnosed cancers worldwide and the third leading cause of cancer-related mortality after lung and liver cancer (Ferlay et al. 2015). The carcinogenesis and progression of GC is complex, involving the alternations of multi-step and multi-genes (Zhao et al. 2017). The 5-year survival rate of GC diagnosed at a later stage is less than $20 \%$, but rises to $90 \%$ for patients diagnosed at an early stage (Stahl et al. 2017). Although traditional biomarkers including carbohydrate antigen 19-9 (CA 19-9) and carcino-embryonic antigen (CEA) have improved clinical outcomes for GC, their sensitivity and specificity are still limited (Ding et al. 2017). Therefore, the identification of novel screening biomarkers may help better diagnose and improve the prognosis of GC (Stahl et al. 2017). 
64 Recently, there is a growing interest in circulating messenger RNAs (mRNAs) isolated from 65 body fluids as potential minimally invasive biomarkers for cancer detection (Kishikawa et al. 66 2015; Sole et al. 2019). Numerous studies have reported that circulating mRNAs can be detected 67 in GC (Funaki et al. 1996), melanoma (Kopreski et al. 1999) and nasopharyngeal carcinoma (Lo 68 et al. 1999). These discoveries provide evidence for circulating mRNAs to be served as 69 appealing non-invasive biomarker candidates in various cancers. However, few circulating 70 mRNAs have been investigated in GC and limited studies have compared the expression level of 71 circulating mRNAs in plasma of GC patients with the clinicopathological characteristics.

72 The establishment of high-throughput molecular database such as microarray databases brings a 73 new approach for biomarker identification. We have previously published an effective 74 bioinformatics scheme to identify noninvasive biomarkers for lung cancer (Zhou et al. 2017). In 75 the present study, we applied the similar approach to explore mRNAs circulated in plasma from 76 patients with GC as potential biomarkers. Subsequently, the associations between these 77 biomarkers and clinicopathological characteristics were analyzed. Finally, the relationships 78 between these selected noninvasive biomarkers and overall survival (OS) were investigated.

\section{Materials \& Methods}

\section{Genome-wide expression analysis by Oncomine}

82

83

84

85

86

87

88

89

90

91

92

93

94

95

96

97

98

99

Expression profiling including $304 \mathrm{GC}$ cancer samples and 174 normal controls was obtained from Oncomine microarray database (www.oncomine.org) (Rhodes et al. 2004). In order to analyze the expression pattern of cancer vs. normal tissue mRNA, we focused on primary tumors and the following cut-offs were employed $p$-value $\leq 10^{-4}$, fold change $\geq 2$ and gene rank $\leq 10 \%$. Heat maps of overexpressed and underexpressed mRNAs in GC were available for each study.

\section{Clinical specimens}

Peripheral blood samples from 56 patients with gastric adenocarcinoma were addressed before therapeutic intervention by venipuncture and processed within $2 \mathrm{hr}$ at the First Affiliated Hospital of Xiamen University. We also collected blood samples from 14 healthy volunteers. The healthy volunteers presented with neither history of cancer nor other diseases. All patients were pathologically diagnosed as having gastric cancer using surgical specimens and biopsies. Plasma was isolated from $4 \mathrm{ml}$ blood specimens after centrifugation at $1,600 \times \mathrm{g}$ for $10 \mathrm{~min}$ at $4^{\circ} \mathrm{C}$ and $10,000 \times \mathrm{g}$ for $10 \mathrm{~min}$, and then stored at $-80^{\circ} \mathrm{C}$ until the next step. Demographic, clinical and histopathological parameters of all these cases were summarized in Table 1. All experimental protocols were approved by the Clinical Research Ethics Committee of the First Affiliated Hospital of Xiamen University. All methods were performed in accordance with the Declaration of Helsinki. Written informed consent was obtained from all human participants after complete description of the study (Fig. S1 and Table. S2). 
100

101

102

103

104

105

106

107

108

109

110

111

112

113

114

115

116

117

118

119

120

121

122

123

124

125

126

127

128

129

130

131

132

133

134

135

\section{RNA extraction}

RNA was extracted from 500 $\mu$ l plasma using TRIzol LS reagent (cat\#10296018, Thermo Fisher Scientific Inc.) following manufacturer's instructions as previously described (Pucciarelli et al. 2012; Zhang et al. 2012). In brief, $500 \mu$ l plasma was mixed with $500 \mu l$ TRIzol reagent. After $5 \mathrm{~min}$ incubation at $4^{\circ} \mathrm{C}, 500 \mu \mathrm{l}$ chloroform was added to the mixture, and violently shook for 30 sec. The mixture was immediately centrifuged at $12,000 \times \mathrm{g}$ for $5 \mathrm{~min}$ at $4^{\circ} \mathrm{C}$. The above aqueous layer was transferred into a fresh tube containing $800 \mu$ isopropyl alcohol. Next, the mixture was centrifuged at $12,000 \times \mathrm{g}$ for $5 \mathrm{~min}$ at $4^{\circ} \mathrm{C}$ and washed with $1 \mathrm{ml} 70 \%$ ethanol for twice. Lastly, the RNA pellet was dissolved in $15 \mu \mathrm{l}$ RNase-free water. Qubit RNA HS Assay Kit and Qubit 3.0 fluorometer (ThermoFisher Scientific Inc. cat\#Q32851) were employed to quantify the concentration of RNA solution through spectrophotometry. The concentration of RNA isolated from plasma ranged was $35.24-168.18 \mathrm{ng} / \mu \mathrm{l}$.

\section{Quantitative PCR (qPCR)}

The extracted total RNA was reverse-transcription into cDNA using PrimeScript RT reagent Kit (TAKARA cat\#RR047A) according to the manufacturer's protocol in triplicate. The resulting cDNA was stored at $-80^{\circ} \mathrm{C}$ for next PCR amplification. Primer sequences were designed through web-based version 4.1.0 of Primer 3 and were shown in Table. S1. For clinical validation of the bioinformatics analysis results, qPCR was conducted by ABI ViiA 7 Real-Time PCR System (Applied Biosystems) with melting curve analysis. QPCR was carried out in triplicate at $50^{\circ} \mathrm{C}$ for $2 \mathrm{~min}$, denaturing at $95^{\circ} \mathrm{C}$ for $5 \mathrm{~min}$, followed by 40 cycles at $95^{\circ} \mathrm{C}$ for $30 \mathrm{~s}, 58^{\circ} \mathrm{C}$ for $1 \mathrm{~min}$. Glyceraldehyde-3-phosphate dehydrogenase (GAPDH) was selected as an internal control. Water negative controls contained all components for the qPCR reaction without target RNA. Positive controls of RNA were extracted from SGC7901 cells obtained from the Cancer Center of Xiamen University (Xiamen, China). The $2^{-\Delta C T}$ algorithm $(\Delta C \mathrm{CT}=\mathrm{Ct}$. target-Ct. reference) was employed for data analysis (Maru et al. 2009).

\section{The Kaplan-Meier plotter analysis}

The prognostic value of candidate circulating mRNAs was analyzed using the Kaplan-Meier plotter database, an online database containing 54,675 genes on survival based on $1065 \mathrm{GC}$ samples with a mean follow-up of 33 months (Szasz et al. 2016). Overall survival of patients with high and low expression levels of target genes was displayed using Kaplan-Meier survival curves. Hazard ratios (HR) with 95\% confidence intervals (CI), and log-rank p-values were also calculated and summarized.

\section{Functional enrichment network}

Gene functional network was performed using gene ontology enrichment (GO) analysis. Enrichment map were created using the Cytoscape (v3.6.0). FDR $<0.05$ was considered to be significant. 
137 The Mann-Whitney $U$ test was used to compare the expression status of circulating mRNAs in 138 normal and GC groups and calculate the relationship between clinicopathologic characteristics 139 and expression levels of relevant mRNA. Data was shown as median and range. Receiver 140 operating characteristic (ROC) curves and the area under the curve (AUC) was used to identify the diagnosis value of selected mRNA (Brumback et al. 2006). Cut-off values were assessed at different sensitivities and specificities and at the maximum Youden's index $=($ sensitivity + specificity-1) $\quad($ Youden 1950). Then, the logistic regression model was performed to obtain a combined ROC curve. GraphPad Prism 6.0 (GraphPad Soft-ware Inc., La Jolla, CA) and SPSS (version 22.0, IBM SPSS co., USA) was used for these statistical analyses. A two-sided $p$ value of less than 0.05 was defined as statistically significant.

\section{Results}

\section{Identification of candidate mRNAs from Oncomine database}

150

151

152

153

154

155

156

157

158

159

160

161

162

163

164

165

166

167

168

169

170

171

We compared the mRNA expression level in gastric cancer vs. normal samples obtained from Oncomine database. In total, $304 \mathrm{GC}$ samples including 50 diffuse gastric adenocarcinoma, 21 gastric adenocarcinoma, 113 gastric intestinal type adenocarcinoma, 22 gastric mixed adenocarcinoma, 6 gastrointestinal stromal tumor and 92 other GC and 174 normal controls from 5 selected microarray datasets were analyzed (Chen et al. 2003; Cho et al. 2011; Cui et al. 2011; D'Errico et al. 2009; Wang et al. 2012). As shown in Fig. 1, expression profiling analysis generated 40 altered mRNA expressions in GC. The top 10 overexpressed and underexpressed genes embodied important genes involved in carcinogenesis and progression as well as several uncharacterized candidates (collagen type VI alpha 3 chain (COL6A3), serpin family H member 1 (SERPINH1), pleckstrin homology and RhoGEF domain containing G1 (PLEKHG1), collagen type I alpha 2 chain (COL1A2), claudin 1 (CLDN1), metallopeptidase inhibitor 1 (TIMP1), NOP56 ribonucleoprotein (NOP56), collagen type IV alpha 1 chain (COL4A1), immunoglobulin like domain containing receptor 1 (ILDR1); cadherin 11 (CDH11), pepsinogen 4, group I pepsinogen A (PGA4), potassium voltage-gated channel subfamily E regulatory subunit 2 (KCNE2), gastric intrinsic factor (GIF), solute carrier family 9 member A4 (SLC9A4), estrogen related receptor gamma (ESRRG), ATPase $H+/ K+$ transporting subunit alpha (ATP4A), ATPase $H+/ K+$ transporting subunit beta (ATP4B), PRDM16 divergent transcript (FLJ42875), $M A S$ related GPR family member $D(M R G P R D)$ and tripartite motif containing 50 (TRIM50), respectively).

\section{Experimental validation of the potential noninvasive biomarkers}

To validate our expression profiling analyses, 6 selected genes including top 3 overexpressed (COL6A3, SERPINH1 and PLEKHG1) and underexpressed (PGA4, KCNE2 and GIF) mRNAs 
172 were experimental verified using circulating mRNA extracted from $56 \mathrm{GC}$ patients and 14 173 healthy subjects.

174 The expression of COL6A3, PLEKHG1, PGA4 and KCNE2 was detected in 55/56, 41/56, 32/56

175

176

177

178

179

180

181

182

183

184

185

186

187

188

189

190

191

192

193

194

195

196

197

198

199

200

201

202

203

204

205

206 and 55/56 GC plasma samples respectively, whereas the expression of SERPINH1 and GIF was detected in all GC samples. As shown in Fig. 2, the expression level of COL6A3 $(P=0.0128)$, SERPINHI $(P=0.0217)$ and PLEKHGI $(P=0.0064)$ was significantly higher in GC plasmas than those in normal controls. However, the expression levels of PGA4, KCNE2 and GIF had no significant change.

\section{Diagnostic value of plasma COL6A3, SERPINH1 and PLEKHG1 for GC}

We performed ROC curve analysis to assess the diagnostic value of these three circulating mRNAs (Fig. 3). The plasma level of COL6A3 had a sensitivity of $100 \%$ and a specificity of $46.2 \%$ to differentiate the GC patients from the healthy subjects, with an AUC of 0.720 at the optimal cut-off point $(95 \% \mathrm{CI}$ : $0.522-0.919)$. SERPINH1 had a sensitivity of $58.9 \%$ and a specificity of 78.6\%, with an AUC of 0.698 (95\%CI: 0.543-0.852). PLEKHG1 had a sensitivity of $68.3 \%$ and a specificity of $100 \%$, with an AUC of 0.833 (95\% CI: $0.699-0.968$ ). Combining any two of the biomarkers had values for ROC area ranging from 0.676 to 0.870 , sensitivities from $60 \%$ to $82.9 \%$, specificities from $76.9 \%$ to $100 \%$ (Fig. 4). Furthermore, we used logistic regression analysis to combine these three circulating mRNAs and obtained an increased AUC value of 0.907 (95\%CI: $0.820-0.993$ ), with a sensitivity of $82.9 \%$ and a specificity of $100 \%$.

Associations between clinicopathological characteristics and plasma COL6A3, SERPINH1 and PLEKHG1

The associations between clinicopathological characteristics and these three circulating mRNAs were investigated. As shown in Table. 2, the higher COL6A3 level was significantly associated with increased lymph node metastasis $(P=0.0233)$, whereas the elevated expression of SERPINH1 was associated with advanced age $(P=0.0034)$ and poor differentiation $(P=0.0231)$ in GC patients. No significant association between PLEKHG1 and any clinicopathological characteristics including age, sex, stage, lymph node metastasis or differentiation was found.

\section{Increased COL6A3, SERPINH1 and PLEKHG1 is associated with poor prognosis}

To further evaluate whether the expression levels of COL6A3, SERPINH1 and PLEKHG1 can predict prognosis, we performed a survival analysis based on publicly gene expression datasets from the Kaplan-Meier Plotter resource. As shown in Fig. 5, the higher expression of COL6A3 $(\mathrm{HR}=1.32,95 \% \mathrm{CI}: 1.11-1.58, \mathrm{p}=0.0018)$, SERPINH1 (HR $\left.=1.97,95 \% \mathrm{CI}: 1.61-2.41, \mathrm{p}=3.1 \mathrm{e}^{-11}\right)$ and PLEKHG1 ( $\mathrm{HR}=1.34,95 \% \mathrm{CI}: 1.07-1.69, \mathrm{p}=0.012)$ were all significantly correlated with poor OS in GC. These results indicated that GC patients with high COL6A3, SERPINHI or PLEKHG1 tend to have unfavorable outcome. 


\section{GO functional enrichment analysis}

208 Functional enrichment network of COL6A3, SERPINH1 and PLEKHG1 was constructed. As 209 shown in Fig. 6A, COL6A3 was predicted to have the main functions: extracellular matrix 210 organization, cell adhesion, multicellular organism development, animal organ development and 211 system development. SERPINH1 played roles in extracellular matrix organization, collagen fibril 212 organization, skeletal system development, collagen metabolic process, and animal organ

213 morphogenesis (Fig. 6B). PLEKHG1 was found associated with Rho guanyl-nucleotide 214 exchange factor activity (Fig. 6C).

215

\section{Discussion}

217 The survival of GC affected patients depends mainly on early detection (Wang et al. 2006). The

218 common screening approaches are gastroscopy and computed tomography, which are invasive 219 and expensive (Ke et al. 2017). Therefore, easily accessible and noninvasive biomarkers derived

220 from body fluid are prevalent (Shen et al. 2017). Exploration of circulating biomarkers for

221

222

223

224

225

226

227

228

229

230

231

232

233

234

235

236

237

238

239

240

241

242

243 various cancer types can be conducted through different approaches. In the present study, we identified candidate biomarkers according to our previous strategy which combined comprehensive analysis of microarray data and experimental validation using plasma samples (Zhou et al. 2017). We first identified three circulating mRNA markers (COL6A3, SERPINH1 and $P L E K H G 1$ ) that carry diagnostic potential for GC. We also found that combination of these three markers exhibited better diagnostic performance for $\mathrm{GC}$ than each individual marker. Our strategy can also be flexibly applied to various diseases.

Circulating RNA including lncRNA, mRNA and microRNA can be isolated and detected in serum, plasma, urine and lymph. In bodily fluid, RNA molecules are directly exposed to RNase resulting in the degradation of RNAs and the difficulty to identify RNA based biomarkers (Hasselmann et al. 2001; Sisco 2001). However, some studies have suggested that circulating RNA is especially stable due to the protection from phospholipids (Elhefnawy et al. 2004; Halicka et al. 2000; Ma et al. 2012). In addition, another study demonstrate that the concentration of circulating RNA in GC patients is higher than that of healthy controls, which is associated with tumor growth and metastasis metabolism (Elhefnawy et al. 2004; Rykova et al. 2006). In this study, we found that the levels of plasmatic COL6A3, SERPINH1 and PLEKHG1 were significantly increased in patients with GC than in healthy subjects.

COL6A3, located at chromosome $2 \mathrm{q} 37$, encodes the alpha-3 chain for type VI collagen (Dankel et al. 2014). Collagen VI has been initially defined as an extracellular matrix protein and it is expressed in various tissues such as muscle, skin and cartilage. COL6A3 is a secreted protein and have received growing attention due to its abnormal expression in colon, pancreatic, bladder and prostate cancer (Kang et al. 2014; Thorsen et al. 2008). In a previous study, COL6A3 has been shown to be a potential plasma marker of colorectal cancer and is associated with tumor 
244 metastasis (Qiao et al. 2015). However, the expression pattern and function of COL6A3 in the 245 tumorigenesis of $\mathrm{GC}$ remain unclear. Our present study indicated that COL6A3 was 246 overexpressed in plasma of GC patients and was associated with increased lymph node 247 metastasis.

248 SERPINH1, also known as heat shock protein 47 (HSP47), belongs to the serpin superfamily 249 involving serine proteinase inhibitors (Ito \& Nagata 2016). The location of SERPINH1 is at 250 chromosome 11q13.5, a domain frequently abnormal in various human cancers. Numerous 251

252

253

254

255

256

257 studies have demonstrated that SERPINH1 is overexpressed in various human cancers, including lung cancer, pancreatic cancer, cervical cancer and glioma (Wu et al. 2016; Yamamoto et al. 2013). In addition, serum SERPINH1 has been reported to be used as a possible target for patients with scirrhous gastric cancer. Our results showed that the overexpressed SERPINH1 was associated with advanced age and poor differentiation in plasma from GC patients. These data indicated that SERPINH1 played a critical role in GC, although further studies are necessary to clarify the biological mechanism of SERPINH1 in GC.

258

259

260

261

262

263

264

265

266

267

268

269

270

\section{Conclusions}

272 Accordingly, we have identified potential noninvasive biomarkers for gastric cancer using 273 bioinformatics analysis through public database and verified their value using GC clinical tumor 274 and plasma specimens. COL6A3, SERPINH1 and PLEKHG1 are three prospective biomarkers 275 for GC. The combination of plasma COL6A3, SERPINH1 and PLEKHG1 represent a promising 276 diagnostic method. The clinical samples employed in this study were relatively limited. Hence, 277 large-scale studies should be performed to investigate the clinical significance of COL6A3, 278 SERPINH1 and PLEKHG1 in GC in the future. Moreover, further investigation of their 279
PLEKHG1 contains a Rho guanine nucleotide exchange factor domain and a pleckstrin homology domain. PLEKHG1 acts as a signaling platform in various cells, but the detail functions are not clear. In this study, we found that the plasma level of PLEKHG1 mRNA was significantly increased in GC patients compared with normal subjects, with a markedly high AUC value of 0.8333 . These results suggested that PLEKHG1 mRNA have a high diagnosis capability.

We carried out the ROC curve to analyze the diagnostic value of COL6A3, SERPINH1 and PLEKHG1 in plasma from GC patients. The results demonstrated that PLEKHG1 had higher diagnostic value for GC than that of COL6A3 or SERPINH1. More powerful diagnostic values were observed when combining these three mRNAs, resulting in an AUC of 0.907. In addition, the prognostic roles of these three potential biomarkers in GC patients were rarely reported and all these biomarkers were correlated with worse OS for patients with GC. 


\section{Acknowledgements}

282 We would like to thank the patients and healthy subjects for consenting to provide material for 283 this study.

References

286 Brumback LC, Pepe MS, and Alonzo TA. 2006. Using the ROC curve for gauging treatment 287 effect in clinical trials. Statistics in Medicine 25:575-590. 10.1002/sim.2345

288 Chen X, Leung SY, Yuen ST, Chu KM, Ji J, Li R, Chan AS, Law S, Troyanskaya OG, Wong J, 289 So S, Botstein D, and Brown PO. 2003. Variation in gene expression patterns in human gastric 290 cancers. Mol Biol Cell 14:3208-3215. 10.1091/mbc.E02-12-0833

291 Cho JY, Lim JY, Cheong JH, Park YY, Yoon SL, Kim SM, Kim SB, Kim H, Hong SW, and 292 Park YN. 2011. Gene expression signature-based prognostic risk score in gastric cancer. Clinical 293 Cancer Research An Official Journal of the American Association for Cancer Research 17:1850294 1857. 10.1158/1078-0432.CCR-10-2180

295 Cui J, Chen Y, Chou WC, Sun L, Chen L, Suo J, Ni Z, Zhang M, Kong X, Hoffman LL, Kang J, 296 Su Y, Olman V, Johnson D, Tench DW, Amster IJ, Orlando R, Puett D, Li F, and Xu Y. 2011. 297 An integrated transcriptomic and computational analysis for biomarker identification in gastric 298 cancer. Nucleic Acids Res 39:1197-1207. 10.1093/nar/gkq960

299 D'Errico M, de Rinaldis E, Blasi MF, Viti V, Falchetti M, Calcagnile A, Sera F, Saieva C, Ottini 300 L, Palli D, Palombo F, Giuliani A, and Dogliotti E. 2009. Genome-wide expression profile of 301 sporadic gastric cancers with microsatellite instability. Eur J Cancer 45:461-469. 302 10.1016/j.ejca.2008.10.032

303 Dankel SN, Svard J, Mattha S, Claussnitzer M, Kloting N, Glunk V, Fandalyuk Z, Grytten E, 304 Solsvik MH, Nielsen HJ, Busch C, Hauner H, Bluher M, Skurk T, Sagen JV, and Mellgren G. 305 2014. COL6A3 expression in adipocytes associates with insulin resistance and depends on 306 PPARgamma and adipocyte size. Obesity (Silver Spring) 22:1807-1813. 10.1002/oby.20758

307 Ding D, Song Y, Yao Y, and Zhang S. 2017. Preoperative serum macrophage activated 308 biomarkers soluble mannose receptor (sMR) and soluble haemoglobin scavenger receptor 309 (sCD163), as novel markers for the diagnosis and prognosis of gastric cancer. Oncology Letters 310 14:2982-2990. 10.3892/ol.2017.6547 
311 Elhefnawy T, Raja S, Kelly L, Bigbee WL, Kirkwood JM, Luketich JD, and Godfrey TE. 2004. 312 Characterization of amplifiable, circulating RNA in plasma and its potential as a tool for cancer 313 diagnostics. Clin Chem 50:564-573. 10.1373/clinchem.2003.028506

314 Ferlay J, Soerjomataram I, Dikshit R, Eser S, Mathers C, Rebelo M, Parkin DM, Forman D, and 315 Bray F. 2015. Cancer incidence and mortality worldwide: sources, methods and major patterns in 316 GLOBOCAN 2012. Int J Cancer 136:E359-E386. 10.1002/ijc.29210

317 Funaki NO, Tanaka J, Kasamatsu T, Ohshio G, Hosotani R, Okino T, and Imamura M. 1996. 318 Identification of carcinoembryonic antigen mRNA in circulating peripheral blood of pancreatic 319 carcinoma and gastric carcinoma patients. Life Sciences 59:2187-2199. 10.1016/S0024$3203205(96) 00576-0$

321 Halicka HD, Bedner E, and Darzynkiewicz Z. 2000. Segregation of RNA and Separate 322 Packaging of DNA and RNA in Apoptotic Bodies during Apoptosis. Exp Cell Res 260:248-256. 323 10.1006/excr.2000.5027

324 Hasselmann DO, Rappl G, Tilgen W, and Reinhold U. 2001. Extracellular Tyrosinase mRNA 325 within Apoptotic Bodies Is Protected from Degradation in Human Serum. Clin Chem 47:1488326 1489. 10.1016/S0009-8981(01)00527-7

327 Ito S, and Nagata K. 2016. Biology of Hsp47 (Serpin H1), a collagen-specific molecular 328 chaperone. Seminars in Cell \& Developmental Biology 62:142-151. 329 10.1016/j.semcdb.2016.11.005

330 Kang CY, Wang J, Axell-House D, Soni P, Chu ML, Chipitsyna G, Sarosiek K, Sendecki J, 331 Hyslop T, Al-Zoubi M, Yeo CJ, and Arafat HA. 2014. Clinical significance of serum COL6A3 in 332 pancreatic ductal adenocarcinoma. J Gastrointest Surg 18:7-15. 10.1007/s11605-013-2326-y

333 Ke D, Li H, Zhang Y, An Y, Fu H, Fang X, and Zheng X. 2017. The combination of circulating 334 long noncoding RNAs AK001058, INHBA-AS1, MIR4435-2HG, and CEBPA-AS1 fragments in 335 plasma serve as diagnostic markers for gastric cancer. Oncotarget 8:21516-21525. 336 10.18632/oncotarget.15628

337 Kishikawa T, Otsuka M, Ohno M, Yoshikawa T, Takata A, and Koike K. 2015. Circulating 338 RNAs as new biomarkers for detecting pancreatic cancer. World J Gastroenterol 21:8527-8540. 339 10.3748/wjg.v21.i28.8527

340 Kopreski MS, Benko FA, Kwak LW, and Gocke CD. 1999. Detection of Tumor Messenger 341 RNA in the Serum of Patients with Malignant Melanoma. Clinical Cancer Research An Official 342 Journal of the American Association for Cancer Research 5:1961-1965. 
343 Lo KW, Lo YM, Leung SF, Tsang YS, Chan LY, Johnson PJ, Hjelm NM, Lee JC, and Huang 344 DP. 1999. Analysis of Cell-Free Epstein-Barr Virus Associated Rna in the Plasma of Patients 345 with Nasopharyngeal Carcinoma. Clin Chem 45:1292-1294.

346 Ma R, Tao J, and Kang X. 2012. Circulating microRNAs in cancer: origin, function and 347 application. J Exp Clin Cancer Res 31:38-46. 10.1186/1756-9966-31-38

348 Maru DM, Singh RR, Hannah C, Albarracin CT, Li YX, Abraham R, Romans AM, Yao H, 349 Luthra MG, and Anandasabapathy S. 2009. MicroRNA-196a is a potential marker of progression 350 during Barrett's metaplasia-dysplasia-invasive adenocarcinoma sequence in esophagus. 351 American Journal of Pathology 174:1940-1948. 10.2353/ajpath.2009.080718

352 Pucciarelli S, Rampazzo E, Briarava M, Maretto I, Agostini M, Digito M, Keppel S, Friso ML, 353 Lonardi S, and Paoli AD. 2012. Telomere-Specific Reverse Transcriptase (hTERT) and Cell-free 354 RNA in Plasma as Predictors of Pathologic Tumor Response in Rectal Cancer Patients Receiving 355 Neoadjuvant Chemoradiotherapy. Annals of Surgical Oncology 19:3089-3096. 10.1245/s10434$356 \quad 012-2272-\mathrm{z}$

357 Qiao J, Fang C-Y, Chen S-X, Wang X-Q, Cui S-J, Liu X-H, Jiang Y-H, Wang J, Zhang Y, Yang 358 P-Y, and Liu F. 2015. Stroma derived COL6A3 is a potential prognosis marker of colorectal 359 carcinoma revealed by quantitative proteomics. Oncotarget 6:29929-29946. $360 \quad 10.18632 /$ oncotarget.4966

361 Rhodes DR, Yu J, Shanker K, Deshpande N, Varambally R, Ghosh D, Barrette T, Pandey A, and 362 Chinnaiyan AM. 2004. ONCOMINE: a cancer microarray database and integrated data-mining 363 platform. Neoplasia 6:1-6. 10.1016/S1476-5586(04)80047-2

364 Rykova EY, Wunsche W, Brizgunova OE, Skvortsova TE, Tamkovich SN, Senin IS, Laktionov 365 PP, Sczakiel G, and Vlassov VV. 2006. Concentrations of Circulating RNA from Healthy 366 Donors and Cancer Patients Estimated by Different Methods. Annals of the New York Academy 367 of Sciences 1075:328-333. 10.1196/annals.1368.044

368 Shen J, Kong W, Wu Y, Ren H, Wei J, Yang Y, Yang Y, Yu L, Guan W, and Liu B. 2017. 369 Plasma mRNA as liquid biopsy predicts chemo-sensitivity in advanced gastric cancer patients. $\mathrm{J}$ 370 Cancer 8:434-442. 10.7150/jca. 17369

371 Sisco KL. 2001. Is RNA in serum bound to nucleoprotein complexes? Clin Chem 47:1744.

372 Sole C, Arnaiz E, Manterola L, Otaegui D, and Lawrie CH. 2019. The circulating transcriptome 373 as a source of cancer liquid biopsy biomarkers. Semin Cancer Biol. 374 10.1016/j.semcancer.2019.01.003 
375 Stahl D, Braun M, Gentles AJ, Lingohr P, Walter4 A, Kristiansen G, and Gütgemann1 I. 2017. 376 Low BUB1 expression is an adverse prognostic marker in gastric adenocarcinoma. Oncotarget 8. 377 10.18632/oncotarget.19357

378 Szasz AM, Lanczky A, Nagy A, Forster S, Hark K, Green JE, Boussioutas A, Busuttil R, Szabo 379 A, and Gyorffy B. 2016. Cross-validation of survival associated biomarkers in gastric cancer 380 using transcriptomic data of 1,065 patients. Oncotarget 7:49322-49333. 381 10.18632/oncotarget.10337

382 Thorsen K, Sorensen KD, Brems-Eskildsen AS, Modin C, Gaustadnes M, Hein AM, Kruhoffer 383 M, Laurberg S, Borre M, Wang K, Brunak S, Krainer AR, Torring N, Dyrskjot L, Andersen CL, 384 and Orntoft TF. 2008. Alternative splicing in colon, bladder, and prostate cancer identified by 385 exon array analysis. Mol Cell Proteomics 7:1214-1224. 10.1074/mcp.M700590-MCP200

386 Wang L, Zhu J-S, Song M-Q, Chen G-Q, and Chen J-L. 2006. Comparison of gene expression 387 profiles between primary tumor and metastatic lesions in gastric cancer patients using laser

388 389

390

391

392

393

394

395

396

397

398

399

400

401

402

403

404

405

406

407 microdissection and cDNA microarray. World Journal of Gastroenterology 12:6949-6954. 10.1186/1471-230X-6-36

Wang Q, Wen YG, Li DP, Xia J, Zhou CZ, Yan DW, Tang HM, and Peng ZH. 2012. Upregulated INHBA expression is associated with poor survival in gastric cancer. Med Oncol 29:77-83. 10.1007/s12032-010-9766-y

Wu ZB, Cai L, Lin SJ, Leng ZG, Guo YH, Yang WL, Chu YW, Yang SH, and Zhao WG. 2016. Heat Shock Protein 47 Promotes Glioma Angiogenesis. Brain Pathol 26:31-42. 10.1111/bpa.12256

Yamamoto N, Kinoshita T, Nohata N, Yoshino H, Itesako T, Fujimura L, Mitsuhashi A, Usui H, Enokida H, Nakagawa M, Shozu M, and Seki N. 2013. Tumor-suppressive microRNA-29a inhibits cancer cell migration and invasion via targeting HSP47 in cervical squamous cell carcinoma. Int J Oncol 43:1855-1863. 10.3892/ijo.2013.2145

Youden WJ. 1950. Index for rating diagnostic tests. Cancer 3:32-35. 10.1002/10970142(1950)3:1<32::aid-cncr2820030106>3.0.co;2-3

Zhang X, Wang C, Wang L, Du L, Wang S, Zheng G, Li W, Zhuang X, Zhang X, and Dong Z. 2012. Detection of circulating Bmi-1 mRNA in plasma and its potential diagnostic and prognostic value for uterine cervical cancer. Int J Cancer 131:165-172. 10.1002/ijc.26360

Zhao H, Wen J, Dong X, He R, Gao C, Zhang W, Zhang Z, and Shen L. 2017. Identification of AQP3 and CD24 as biomarkers for carcinogenesis of gastric intestinal metaplasia. Oncotarget. 10.18632/oncotarget. 18817

Peer] reviewing PDF | (2018:12:33244:1:1:NEW 5 Apr 2019) 
408 Zhou D, Tang W, Xinli Liu1, An H-X, and Zhang Y. 2017. Clinical verification of plasma 409 messenger RNA as novel noninvasive biomarker identified through bioinformatics analysis for 410 lung cancer. Oncotarget. 10.18632/oncotarget.16701

411 


\section{Figure 1}

Transcriptional heat map of the top 20 over- and underexpressed genes in gastric cancer samples compared with normal samples through Oncomine analysis.

The level plots depict the frequencies (\%) of (A) over- and (B) underexpressed candidate messenger RNAs (mRNAs) in 12 analyses from 5 included studies. Red cells represent overexpression. Blue cells represent underexpression. Gray cells represent not measured.

$\mathbf{A}$

Comparison of All Genes Across 12 Analyses

Over-expression

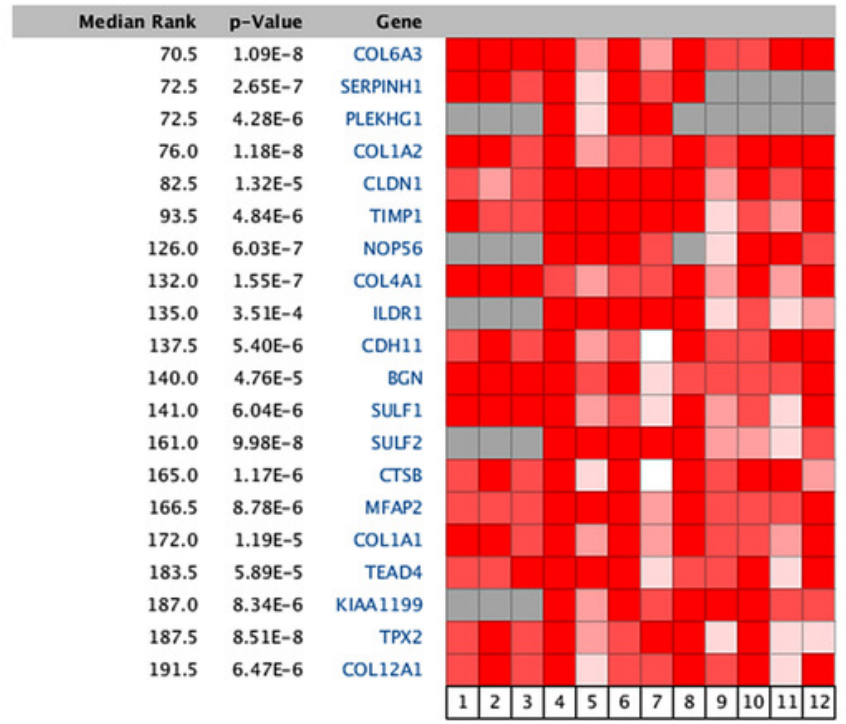

B

\section{Median Rank}

$\begin{array}{rr}\text { p-Value } \\ 4.0 & 1.54 \mathrm{E}-14\end{array}$

$9.0 \quad 2.11 \mathrm{E}-11$

$12.0 \quad 3.00 \mathrm{E}-12$

$14.5 \quad 5.13 \mathrm{E}-10$

$18.0 \quad 1.54 \mathrm{E}-13$

$20.0 \quad 1.11 \mathrm{E}-10$

$22.0 \quad 1.65 \mathrm{E}-10$

$26.0 \quad 1.31 \mathrm{E}-12$

$27.0 \quad 4.17 \mathrm{E}-10$

$28.0 \quad 4.28 \mathrm{E}-10$

$29.0 \quad 6.17 \mathrm{E}-$

$44.0 \quad 2.96 \mathrm{E}-9$

$57.0 \quad 6.89 \mathrm{E}-11$

$68.0 \quad 1.31 \mathrm{E}-8$

$71.0 \quad 1.09 \mathrm{E}-4$

$78.5 \quad 1.32 \mathrm{E}-8$

$81.0 \quad 2.52 \mathrm{E}-7$

$82.0 \quad 4.36 \mathrm{E}-8$

$92.0 \quad 5.32 \mathrm{E}-8$

$97.0 \quad 4.31 \mathrm{E}-7$
All Genes Across 12 Analyses

Under-expression

Gene

PCA4

KCNE2

GIF

SLC9A4

ESRRC

ATP4A

ATP4B

FLJ42875

MRGPRD

TRIM50

GHRL

CWH43

KCNJ16

CCKBR

SH3GL2

KRTAP10-2

APLP1

RDH12

CHCA

AMTN

\section{Legend}

1. Diffuse Gastric Adenocarcinoma vs. Normal 7. Gastric Mixed Adenocarcinoma vs. Normal Chen Gastric, Mol Biol Cell, 2003 Cho Gastric, Clin Cancer Res, 2011

2. Gastric Intestinal Type Adenocarcinoma vs. 8. Gastric Cancer vs. Normal Normal Chen Gastric, Mol Biol Cell, 2003

3. Gastric Mixed Adenocarcinoma vs. Normal Chen Gastric, Mol Biol Cell, 2003

4. Diffuse Gastric Adenocarcinoma vs. Normal Cho Gastric, Clin Cancer Res, 2011

5. Gastric Adenocarcinoma vs. Normal Cho Gastric, Clin Cancer Res, 2011 Cui Gastric, Nucleic Acids Res, 2011

9. Diffuse Gastric Adenocarcinoma vs. Normal DErrico Gastric, Eur J Cancer, 2009

10. Gastric Intestinal Type Adenocarcinoma vs. Normal DErrico Gastric, Eur J Cancer, 2009

6. Gastric Intestinal Type Adenocarcinoma vs. 12. Gastric Cancer vs. Normal Normal Cho Gastric, Clin Cancer Res, 2011 Wang Gastric, Med Oncol, 2010

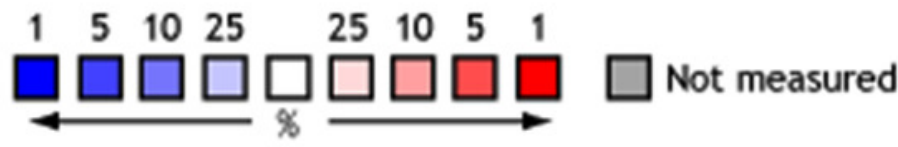

The rank for a gene is the median rank for that gene across each of the analyses. The p-Value for a gene is its p-Value for the median-ranked analysis. 


\section{Figure 2}

Experimental validation of the top 6 over- and underexpressed messenger RNAs (mRNAs).

The change in circulating mRNA levels of collagen type VI alpha 3 chain (COL6A3) (A), serpin family H member 1 (SERPINH1) (B), pleckstrin homology and RhoGEF domain containing G1 (PLEKHG1) (C), pepsinogen 4, group I (pepsinogen A) (PGA4) (D), potassium voltage-gated channel subfamily $E$ regulatory subunit 2 (KCNE2) (E) and gastric intrinsic factor (GIF) (F) between gastric cancer patients and normal subjects detected by qPCR using Student'sttest. And the Mann-Whitney $\mathrm{U}$ test was used to compare the expression status of circulating mRNAs in normal and GC groups. Data was shown as box plots and the intersecting line represents the median value with the interquartile range. Results were shown with means \pm SEM. $* p<0.05, * * p<0.01$. 

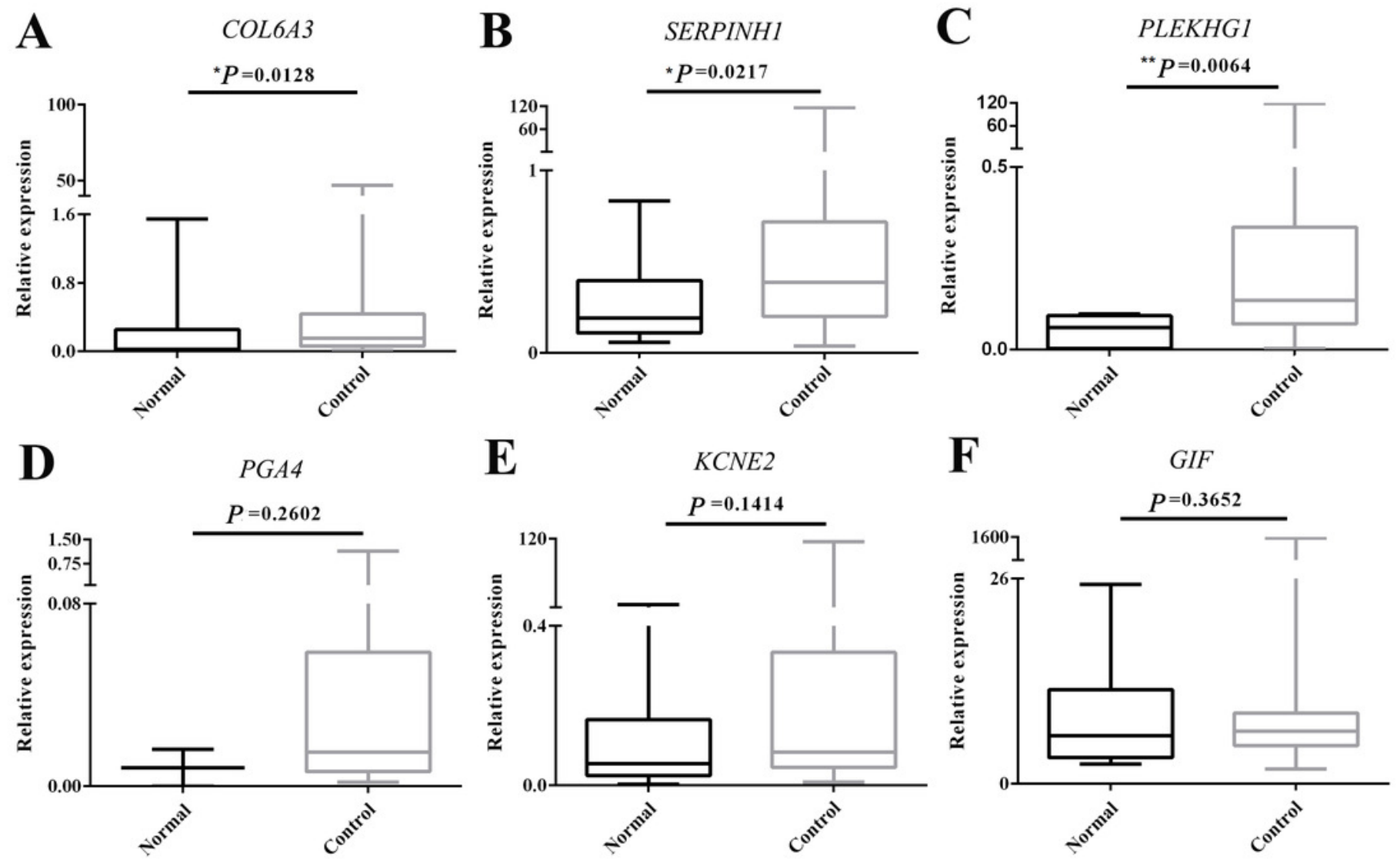
Figure 3

Receiver-operating characteristic curves (ROC) analysis of selected markers in gastric cancer.

The results showed the performances of fold-change in collagen type $\mathrm{VI}$ alpha 3 chain (COL6A3) (A), serpin family H member 1 (SERPINH1) (B) and pleckstrin homology and RhoGEF domain containing GI (PLEKHG1) (C) messenger RNAs (mRNAs) expression in predicting the gastric cancer. ${ }^{*} p<0.05, * * p<0.01$.
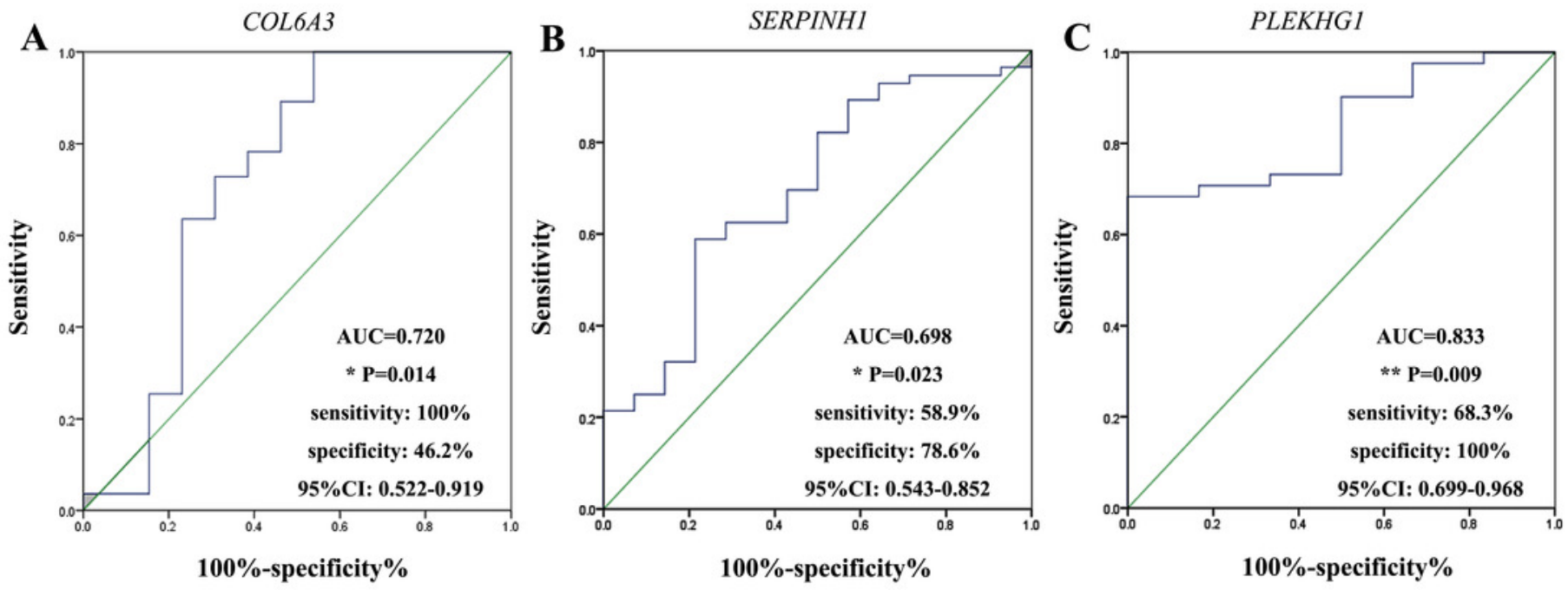


\section{Figure 4}

Receiver-operating characteristic curves (ROC) for combining markers in gastric cancer.

The results showed combination of collagen type VI alpha 3 chain (COL6A3) and pleckstrin homology and RhoGEF domain containing G1 (PLEKHG1) (A), COL6A3 and serpin family $H$ member 1 (SERPINH1) (B), SERPINH1 and PLEKHG1 (C), and combination of these three genes (D) to differentiate patients with gastric from normal subjects. 

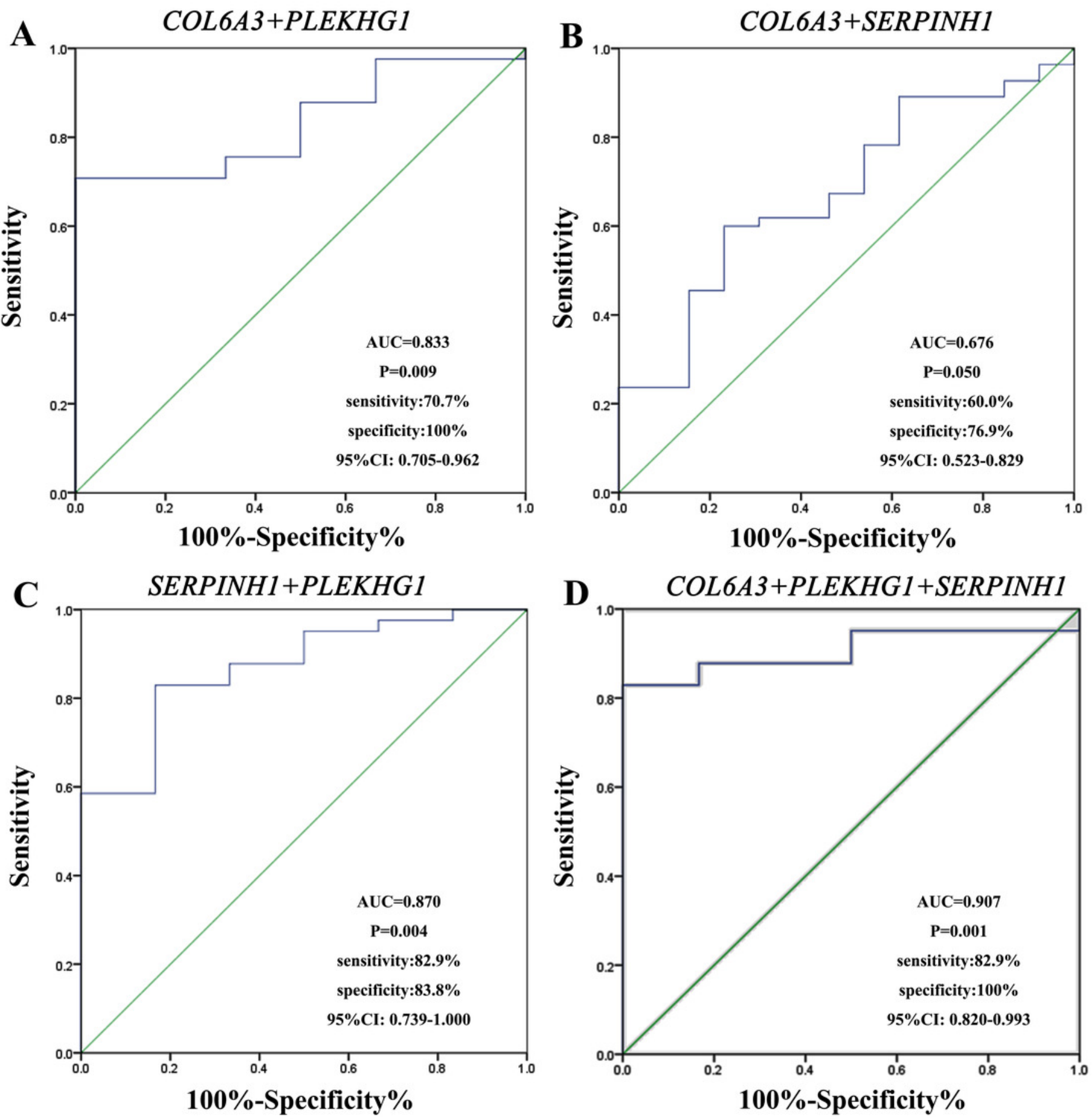
Figure 5

Correlation of collagen type VI alpha 3 chain (COL6A3), serpin family $\mathrm{H}$ member 1 (SERPINH1) and pleckstrin homology and RhoGEF domain containing G1 (PLEKHG1) with survival outcomes in gastric cancer patients.

Increased expression of COL6A3, SERPINH1 and PLEKHG1 predicted worse overall survival (OS) in gastric cancer.
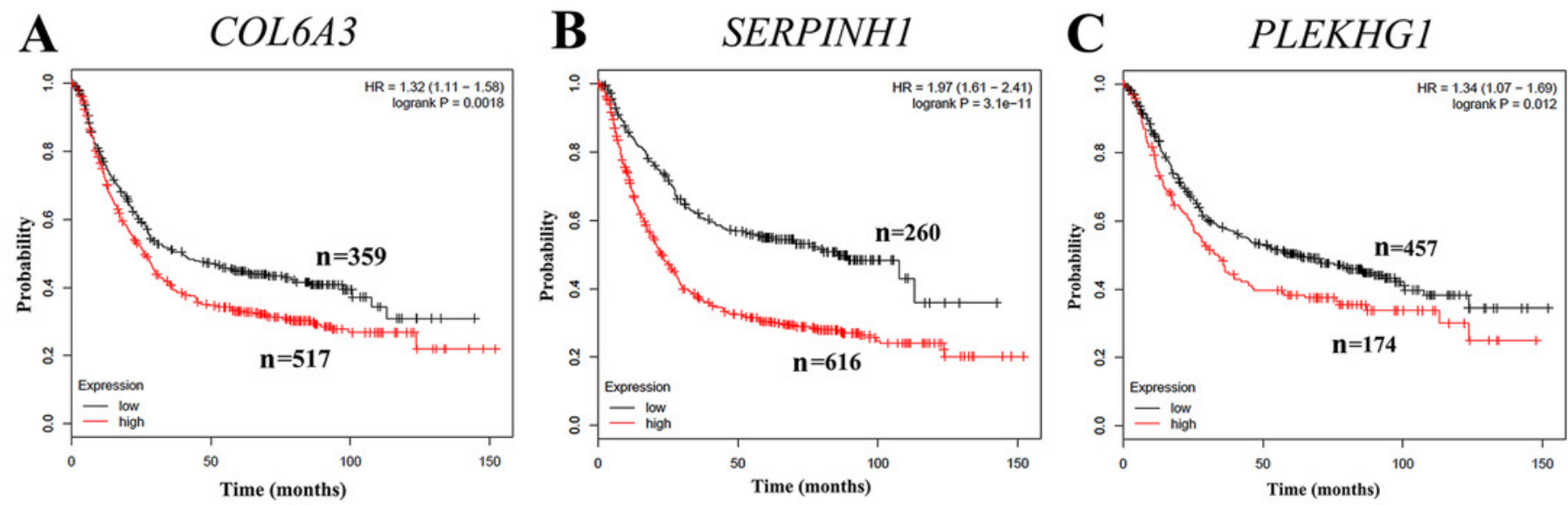
Figure 6

GO functional enrichment analysis of COL6A3 (A), SERPINH1 (B) and PLEKHG1 (C).
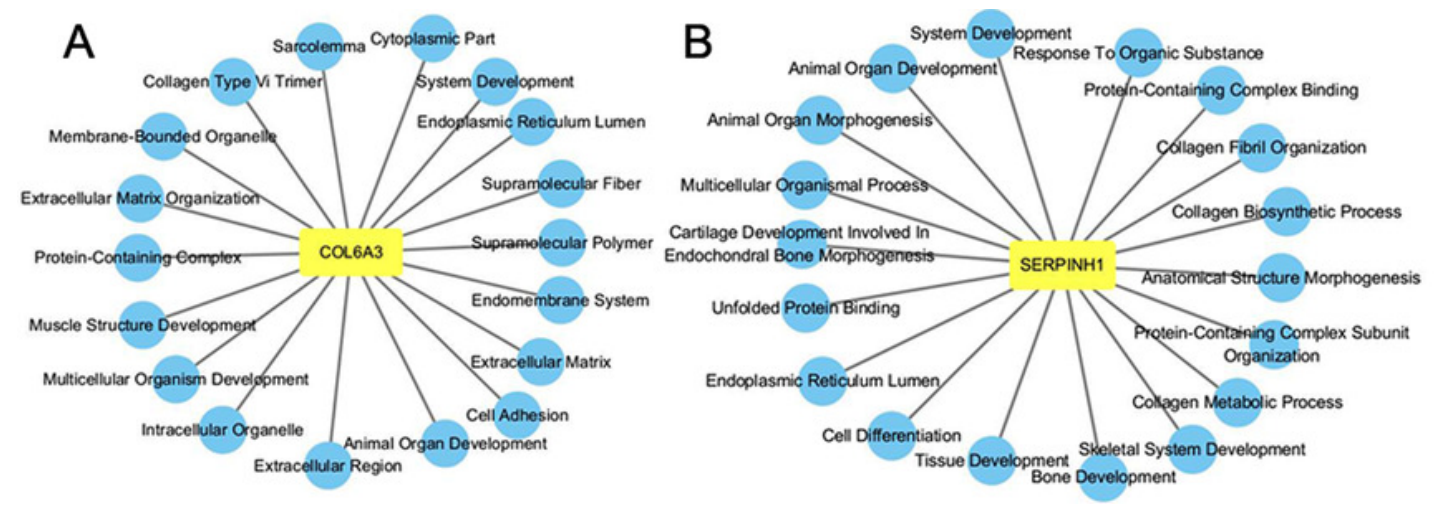

C

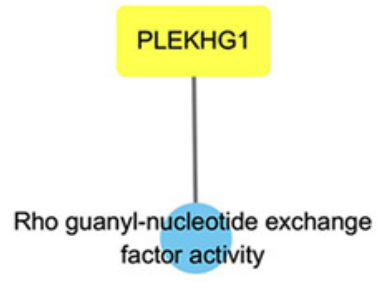




\section{Table 1 (on next page)}

Clinicopathologic characteristics of gastric cancer patients and healthy subjects 
Table 1 Clinicopathologic characteristics of gastric cancer patients

\begin{tabular}{|c|c|c|}
\hline Clinicopathologic factors & Gastric cancer cases & Healthy subjects \\
\hline Total & 56 & 14 \\
\hline Mean Age \pm SD & $62.93 \pm 6.17$ & $43.64 \pm 14.91$ \\
\hline Sex & & \\
\hline Male & 43 & 9 \\
\hline Female & 13 & 5 \\
\hline Stage $^{\mathrm{a}}$ & & \\
\hline $\mathrm{I}+\mathrm{II}+\mathrm{III}$ & 37 & \\
\hline IV & 18 & \\
\hline $\mathrm{N} / \mathrm{A}^{\mathrm{b}}$ & 1 & \\
\hline Lymph node metastasis & & \\
\hline$<15$ & 31 & \\
\hline$\geq 15$ & 7 & \\
\hline
\end{tabular}




\begin{tabular}{|c|c|c|}
\hline N/A & 18 & \\
\hline Differentiation & 19 & \\
\hline Poor & 11 & \\
\hline Moderately or well- & & \\
differentiated & & \\
\hline N/A & 26 & \\
\hline
\end{tabular}

2 aTumor stages were determined according to Union Internationale Contre le Cancer (UICC) 3 criteria.

4 bN/A: not available. 


\section{Table 2 (on next page)}

Associations between the expression of COL6A3, SERPINH1 and PLEKHG1 in gastric cancer and clinicopathologic characteristic. 
1 Table 2 Association between the expression of circulating COL6A3, SERPINH1 and

2 PLEKHG1 in gastric cancer and clinicopathologic characteristics

\begin{tabular}{|c|c|c|c|c|c|c|c|c|c|}
\hline \multirow{2}{*}{ Variable } & \multicolumn{3}{|c|}{ COLGA3 } & \multicolumn{3}{|c|}{ SERPINH1 } & \multicolumn{3}{|c|}{ PLEKHG1 } \\
\hline & $\mathbf{n}$ & Expression status & $\mathbf{P}$ & $\mathbf{n}$ & Expression status & $\mathbf{P}$ & $\mathbf{n}$ & Expression status & $\mathbf{P}$ \\
\hline Age & & & & & & & & & \\
\hline$\square \mathbf{5 0}$ & 12 & $0.179(0.028-1.250)$ & & 12 & $0.190(0.073-1.430)$ & & 10 & $0.130(0.003-1.052)$ & \\
\hline$\geqq \mathbf{5 0}$ & 43 & $\begin{array}{c}0.147(0.011- \\
47.014)\end{array}$ & 0.7091 & 44 & $0.47(0.374-116.407)$ & $0.0034^{* *}$ & 31 & $0.148(0.036-119.46)$ & 0.4517 \\
\hline Sex & & & & & & & & & \\
\hline Male & 42 & $\begin{array}{c}0.150(0.011- \\
10.386)\end{array}$ & & 43 & $0.399(0.374-8.283)$ & & 30 & $0.126(0.036-2.447)$ & \\
\hline Female & 13 & $\begin{array}{c}0.162(0.018- \\
47.014)\end{array}$ & & 13 & $0.325(0.038-116.41)$ & & 11 & $0.161(0.003-119.46)$ & \\
\hline Stage & & & & & & & & & \\
\hline I+II+III & 36 & $\begin{array}{l}0.150(0.011- \\
47.014)\end{array}$ & & 37 & $0.374(0.038-116.41)$ & & 27 & $0.127(0.003-119.46)$ & \\
\hline IV & 18 & $\begin{array}{c}0.190(0.018- \\
10.386)\end{array}$ & & 18 & $0.454(0.118-3.089)$ & & 13 & $0.198(0.065-1.214)$ & \\
\hline Lymph node metastasi & & & & & & & & & \\
\hline$<15$ & 30 & $\begin{array}{c}0.106(0.229- \\
47.014)\end{array}$ & $0.0233^{*}$ & 31 & $0.374(0.221-116.41)$ & 0.6848 & 20 & $0.118(0.036-119.46)$ & 0.6207 \\
\hline$\geq 15$ & 7 & $0.225(0.155-0.698)$ & & 7 & $0.472(0.039-3.623)$ & & 7 & $0.116(0.037-0.336)$ & \\
\hline Differentiation & & & & & & & & & \\
\hline Poor & 19 & $0.198(0.011-47.01)$ & & 19 & $0.362(0.127-116.41)$ & & 12 & $0.110(0.003-119.46)$ & \\
\hline $\begin{array}{c}\text { Moderately or well- } \\
\text { differentiated }\end{array}$ & 11 & $0.033(0.018-0.693)$ & 0.881 & 11 & $0.678(0.159-3.089)$ & $0.0231^{*}$ & 10 & $0.106(0.048-2.447)$ & 0.1059 \\
\hline
\end{tabular}


$4 * *$ : P value $<0.01$

5

Peer] reviewing PDF | (2018:12:33244:1:1:NEW 5 Apr 2019) 